\title{
Serratia odorifera
}

National Cancer Institute

\section{Source}

National Cancer Institute. Serratia odorifera. NCI Thesaurus. Code C86739.

A species of facultatively anaerobic, Gram negative, rod shaped bacteria in the phylum Proteobacteria. This species is motile, peritrichous, positive for both citrase and ornithine decarboxylase, negative for both esterase and lysine decarboxylase and can support growth on and produce acid from arabinose, melibiose, rhamnose, sorbitol, and xylose. S. odorifera can be pathogenic in immunocompromised individuals and symptoms of infection can range from mild signs to severe sepsis and death. 